\title{
Slow Recovery from Critical Coronavirus Disease 2019 Pneumonia in an Immunosuppressed Renal Transplant Recipient with Early Acute Cardiorenal Syndrome
}

\author{
Lan Zhu ${ }^{a, b}$ Hongge Shu ${ }^{c}$ Huijun Lid Tao Qiue Jiangqiao Zhou ${ }^{\text {e }}$ \\ Gang Chen ${ }^{a, b}$ \\ a Institute of Organ Transplantation, Tongji Hospital, Tongji Medical College, Huazhong \\ University of Science and Technology, Wuhan, China; ${ }^{b}$ Key Laboratory of Organ \\ Transplantation, Ministry of Education, Ministry of Public Health, Chinese Academy of Medical \\ Sciences, Wuhan, China; ' Department of Radiology, Tongji Hospital, Tongji Medical College, \\ Huazhong University of Science and Technology, Wuhan, China; ${ }^{\mathrm{d}}$ Department of Laboratory \\ Medicine, Tongji Hospital, Tongji Medical College, Huazhong University of Science and

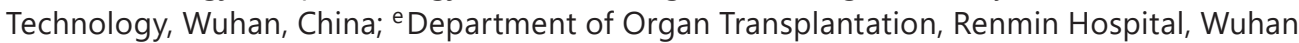 \\ University, Wuhan, China
}

\section{Keywords}

COVID-19 · Renal transplantation · Cardiorenal syndrome - Virus shedding ·

Immunosuppression

\begin{abstract}
With the global spread of SARS-Cov-2 infections, increasing numbers of COVID-19 cases have been reported in transplant recipients. However, reports are lacking concerning the treatment and prognosis of COVID-19 pneumonia in renal transplant recipients with acute cardiorenal syndrome. We report here the complete clinical course of a renal transplant recipient with critical COVID-19 pneumonia. In the early phase of SARS-Cov-2 infection, the patient exhibited extensive lung lesions and significant acute kidney and heart injuries, which required treatment in the ICU. After correcting the arrhythmia and heart failure, the patient recovered quickly from the acute kidney injury with a treatment of intensive diuresis and strict control of fluid intake. Without cessation of oral immunosuppressive agents, the patient presented a delayed and low antibody response against SARS-Cov-2 and reappeared positive for the virus twice after being discharged. Nevertheless, the patient's pneumonia continued to improve and he fully recovered in 69 days. This effectively treated case may be meaningful and referable for the treatment of COVID-19 pneumonia in other transplant recipients with acute cardiorenal syndrome.
\end{abstract}

Gang Chen

Institute of Organ Transplantation, Tongji Hospital

Jiefang Ave. No. 1095

Wuhan 430060 (China)

gchen@tjh.tjmu.edu.cn
Jiangqiao Zhou

Department of Organ Transplantation, Renmin Hospital, Wuhan University Jiefang Rd. No. 238

Wuhan 430060 (China)

zhoujq@whu.edu.cn

\section{Karger}




\section{Cardiorenal Medicine}

\begin{tabular}{l|l}
\hline Cardiorenal Med 2020;10:470-475 \\
\hline DOI: 10.1159/000510916 & $\begin{array}{l}\text { @ 2020 S. Karger AG, Basel } \\
\text { www.karger.com/crm }\end{array}$ \\
\hline
\end{tabular}

Zhu et al.: Recovery from COVID-19 in a Renal Transplant Recipient with Acute Cardiorenal Syndrome

\section{Introduction}

It has been more than 4 months since the outbreak of coronavirus disease 2019 (COVID-19), and increasing numbers of COVID-19 cases have been reported in transplant recipients [1]. As a population living with immunosuppression, renal transplant recipients with SARS-Cov-2 infection have more severe pneumonia compared to the general population [2]. Moreover, the development of acute cardiorenal syndrome in kidney transplant recipients during COVID-19 pneumonia may further increase the difficulty of treatment and patient mortality. However, no relevant reports have as yet been published. In addition, reports are also lacking concerning sequential monitoring of viral nucleic acids and virusspecific antibodies in immunosuppressed transplant recipients with COVID-19 during hospitalization and after discharge. Of the 10 cases of COVID-19 pneumonia in renal transplant recipients we have reported [2], 1 developed severe acute cardiorenal syndrome in the early phase. We further observed the recovery of pneumonia and monitored viral nucleic acids and virus-specific antibodies in this critical patient after discharge, and we report here the detailed clinical features and treatment during the entire course of the disease.

\section{Case Report}

A 55-year-old male patient received a kidney transplant 6 years ago. The kidney graft was obtained from a brain-dead, 26-year-old, male donor whose cause of death was brain trauma. After transplant, he received triple maintenance immunosuppressive therapy with oral tacrolimus (Tac), mycophenolate mofetil (MMF), and methylprednisolone (MP). His renal graft function remained normal. The patient had a history of hypertension for 8 years and coronary heart disease for 1 year.

The patient experienced a decreased urine output, fatigue, and mild cough on February 8, 2020 (the first day of illness). The next day, he went to a local hospital for a chest computed tomography (CT) scan, which revealed significant pneumonia lesions (Fig. 1a). The patient was given oral moxifloxacin ( $0.4 \mathrm{~g}$ qd) and oseltamivir (75 mg q12h). In addition, his daily dose of oral MP was increased from 4 to $24 \mathrm{mg}$, while the doses of Tac and MMF remained unchanged (Fig. 2). On day 4 of illness, the result of a reverse transcription polymerase chain reaction (RT-PCR) assay for SARS-Cov-2 from a pharyngeal swab was positive. The patient was advised to be isolated at home.

On day 6 of illness, the patient was admitted to the intensive care unit (ICU) of our hospital due to chest tightness and aggravation of the oliguria $(<500 \mathrm{~mL} /$ day). Upon admission, the patient exhibited a blood pressure of $90 / 60 \mathrm{~mm} \mathrm{Hg}$, a pulse of 104 beats/min, a respiratory rate of 28 breaths/min, and an oxygen saturation of $94 \%$. Oxygen was then administered to the patient at $5 \mathrm{~L} / \mathrm{min}$ via a nasal catheter. The initial laboratory findings showed a decreased lymphocyte count $\left(0.39 \times 10^{9} / \mathrm{L}\right)$, as well as increased C-reactive protein (CRP; $81.6 \mathrm{mg} / \mathrm{L}$ ), serum creatinine (SCr; $233 \mu \mathrm{mol} / \mathrm{L}$ ), and blood urea nitrogen (BUN; $40.3 \mathrm{mmol} / \mathrm{L}$ ) (Fig. 1b). Two days later, the patient developed shortness of breath, precordial pain, and hemoptysis. His blood pressure $(75 / 55 \mathrm{~mm} \mathrm{Hg})$ and oxygen saturation $(80 \%)$ had decreased further, and his heart rate (120-150 beats/min) and SCr (308 $\mathrm{mol} / \mathrm{L})$ had increased further. An electrocardiographic examination indicated atrial fibrillation. In addition, a sharp rise in serum levels of $\mathrm{N}$-terminal B-type natriuretic peptide (NT-proBNP; $>70,000 \mathrm{pg} / \mathrm{mL}$ ) and cardiac troponin (cTnI; up to 1,580 pg/mL) suggested heart failure and myocardial injury (Fig. 1b). Therefore, the patient was given noninvasive mechanical ventilation to ameliorate the hypoxia, and lanatoside $\mathrm{C}$ and amiodarone were administrated to treat the heart failure and arrhythmia, respectively. The patient was cardioverted to a normal sinus rhythm with a synchronized 90 -J biphasic shock. Subsequently, the patient's blood pressure increased, his urine output increased gradually after intensive diuretic treatment, and his hypoxia was considerably relieved. One day later, the patient was changed to oxygen via a nasal catheter, and his oxygen saturation was maintained at 91-96\%. Other treatments included reduction of the oral MP dose to $8 \mathrm{mg}$ qd, intravenous immunoglobulin (IVIG; $20 \mathrm{~g} /$ day), and strict control of fluid intake. 


\section{Cardiorenal Medicine}

\begin{tabular}{l|l}
\hline Cardiorenal Med 2020;10:470-475 \\
\hline DOI: 10.1159/000510916 & $\begin{array}{l}\text { @ 2020 S. Karger AG, Basel } \\
\text { www.karger.com/crm }\end{array}$ \\
\hline
\end{tabular}

Zhu et al.: Recovery from COVID-19 in a Renal Transplant Recipient with Acute Cardiorenal Syndrome

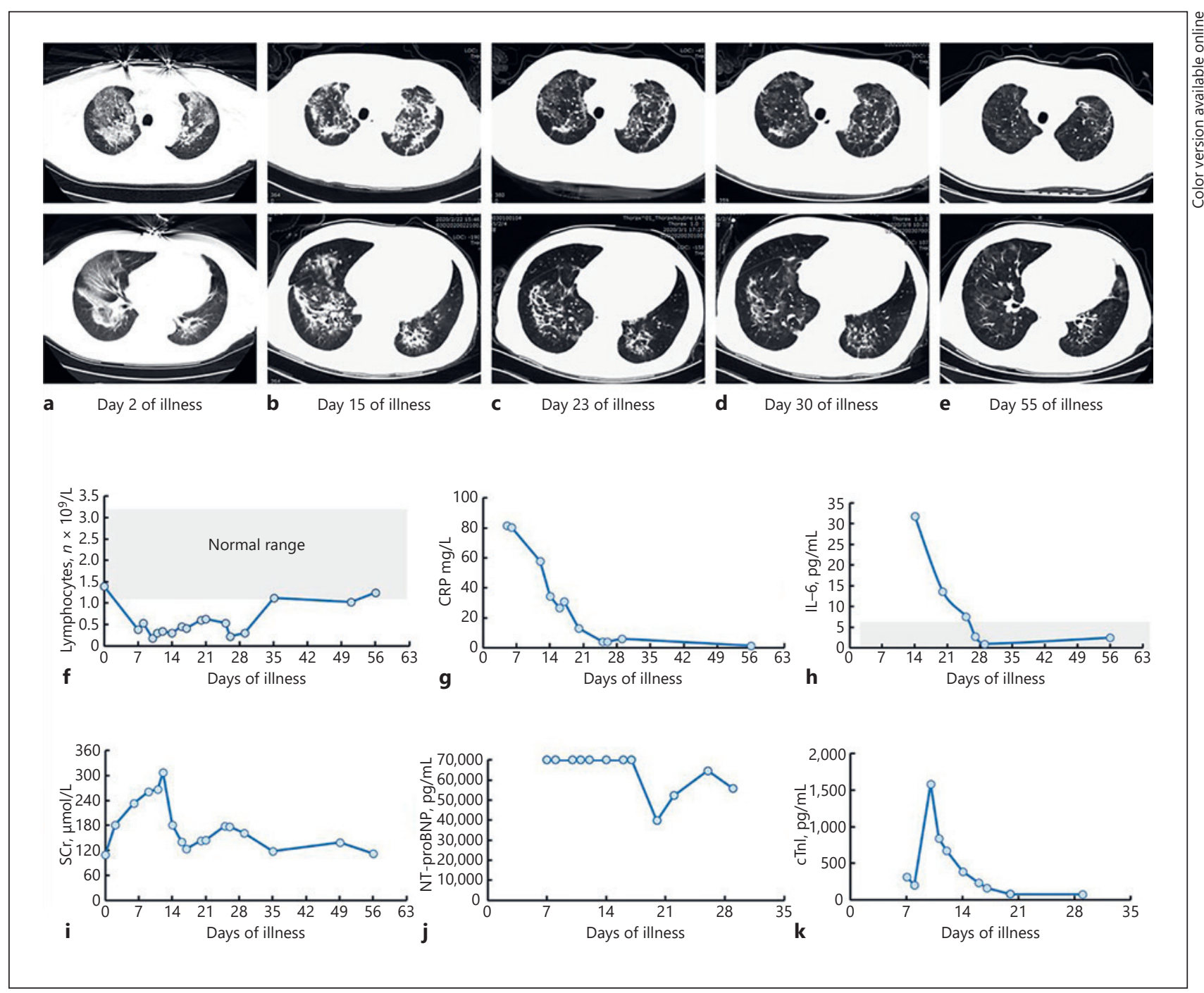

Fig. 1. Changes in radiological (a-e) and laboratory (f-k) results during the entire disease course. Representative chest CT images: on day 2 of illness, multiple bilateral patchy ground-glass opacities and patchy consolidation in the right-side were visible (a); on day 15 of illness, multiple bilateral reticular patterns and increased bilateral patchy consolidation were present (b); on day 23 of illness, the patchy consolidation had nearly disappeared, leaving multiple bilateral reticular patterns (c); on day 30 of illness, the range of multiple bilateral reticular patterns had decreased (d); on day 55 of illness, most of the lesion had been absorbed, and the bilateral reticular patterns had disappeared, leaving a few fibers and blurs (e). Changes in lymphocyte counts (f), CRP (g), IL-6 (c), SCr (h), NT-proBNP (i), and cTnl (j). Reference ranges: lymphocytes, 1.10-

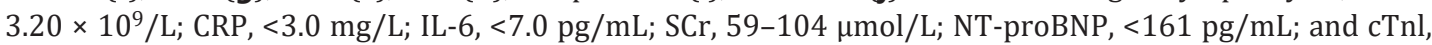
$\leq 34.2 \mathrm{pg} / \mathrm{mL}$.

On day 12 of illness, the patient's respiratory distress was significantly relieved, and the serum levels of CRP, BUN, SCr, and cTnI began to decrease (Fig. 1b). The patient was transferred from the ICU to a general ward and given the following treatment:

- $\quad$ thymosin (1.6 mg intramuscularly, once every 3 days, 3 times in total);

- $\quad$ IVIG (10 g/day for 2 days and $5 \mathrm{~g}$ /day for the next 12 days);

- $\quad$ biapenem (0.3 g i.v. drip q12h for 12 days);

- $\quad$ micafungin (50 mg qd for 10 days); and

- $\quad$ arbidol (also known as umifenovir, orally, $0.2 \mathrm{~g}$ tid for 7 days). 


\section{Medicine \\ Zhu et al.: Recovery from COVID-19 in a Renal Transplant Recipient with Acute Cardiorenal Syndrome}

\begin{tabular}{l|l}
\hline DOI: 10.1159/000510916 & $\begin{array}{l}\text { C 2020 S. Karger AG, Basel } \\
\text { www.karger.com/crm }\end{array}$ \\
\hline
\end{tabular}

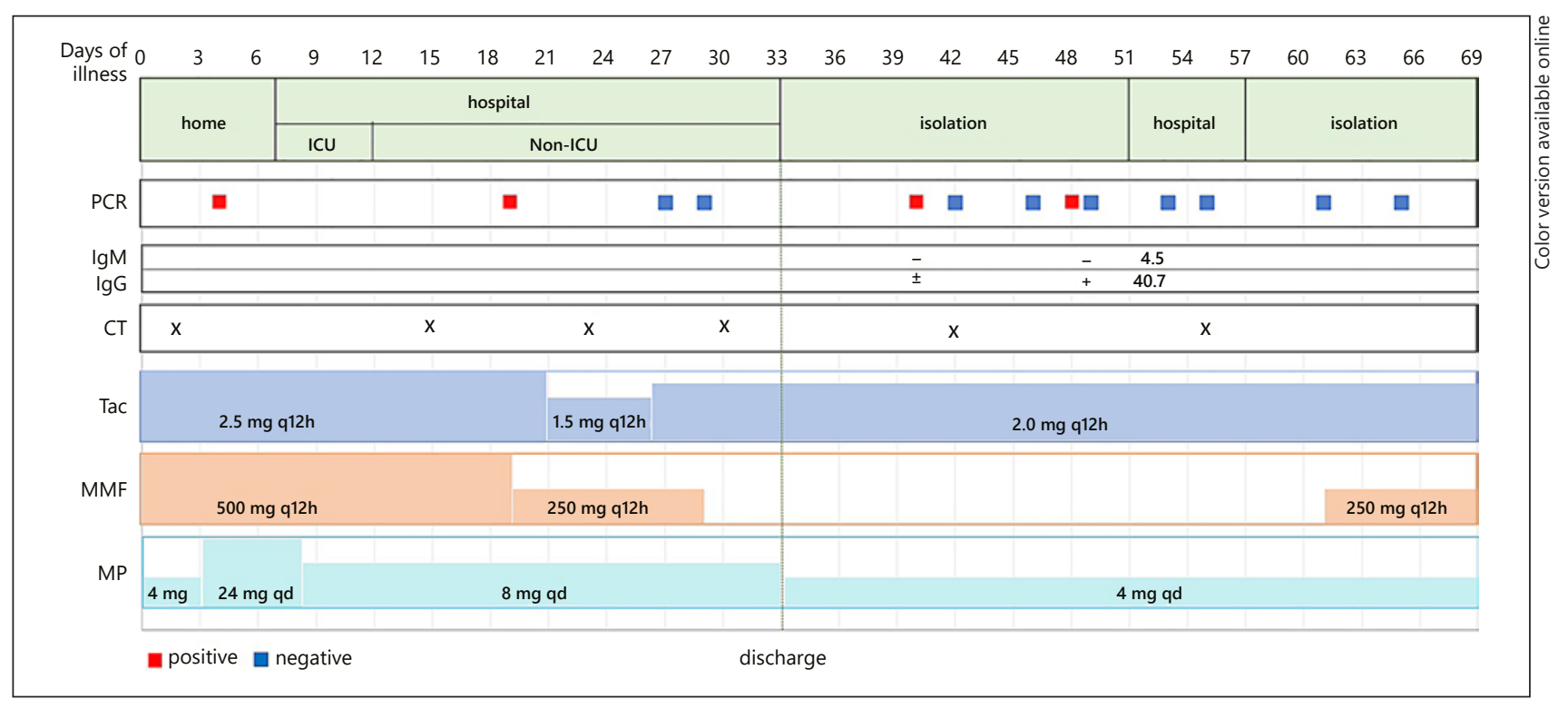

Fig. 2. Results of the detection of SARS-Cov-2 nucleic acid and specific antibodies, time of chest CT examination, and adjustment of immunosuppressive agents throughout the clinical course of the infection. SARSCov-2 antibody levels $<10 \mathrm{AU} / \mathrm{mL}$ are considered negative.

On day 15 of illness, a chest CT scan demonstrated multiple bilateral reticular patterns and increased patchy bilateral consolidation (Fig. 1a, b). The nucleic acid test for SARS-Cov-2 was still positive on day 19 of illness, so reductions in the MMF and Tac dosages were initiated (Fig. 2). From day 27 to day 33 of illness, the levels of CRP and IL-6 decreased to normal (Fig. 1b). More importantly, 2 consecutive throat swab samples tested negative for SARS-Cov-2 (Fig. 2). Meanwhile, the third and fourth chest CT scans showed that the lung lesions were substantially absorbed (Fig. 1a-d). Because of the leukopenia $\left(3.24 \times 10^{9} / \mathrm{L}\right)$, MMF was discontinued on day 29 of illness. On day 33 of illness, the patient was discharged from our hospital.

The patient was then transferred to a designated place for isolation. On day 40 of illness, the results of SARS-Cov-2 nucleic acid were unexpectedly positive for this patient. The results of the qualitative detection of virus-specific antibodies showed that the IgM was negative, and the IgG was weakly positive. Without any additional specific treatment, the test for SARS-Cov- 2 nucleic acid was negative on days 42 and 46 of illness. However, the patient tested positive again on day 48 (Fig. 2). The patient was therefore readmitted to our hospital for observation on day 51 . On day 49 of illness, the results of specific antibodies showed that the IgM was negative and the IgG was positive (Fig. 2). The patient's radiological and laboratory results during his rehospitalization were as follows:

- $\quad$ a chest CT scan revealed further absorption changes of the pneumonia (day 55 of illness) (Fig. 1a-e);

- $\quad$ the trough level of Tac (2 mg, q12h) was $4.8 \mathrm{ng} / \mathrm{mL}$;

- $\quad$ the peripheral blood lymphocyte count returned to normal $\left(1.24 \times 10^{9} / \mathrm{L}\right)$;

- $\quad$ CRP and IL-6 levels remained normal;

- $\quad \mathrm{SCr}$ was $112 \mu \mathrm{mol} / \mathrm{L}$ and BUN was $15 \mathrm{mmol} / \mathrm{L}$;

- $\quad$ SARS-Cov-2-IgM was negative (4.5A U/mL) and SARS-Cov-2-IgG was positive (40.7 AU/mL); and

- $\quad$ SARS-Cov-2 nucleic acid tests were negative on days 53 and 55 of illness.

The patient received no additional special treatment and was discharged after 1 week in the hospital.

After being discharged, the patient was isolated again for 2 weeks, starting on day 57 of illness. During this period, the nucleic acid reexamination for SARS-Cov-2 was negative twice, i.e., on days 61 and 65 of illness, and a low dose of MMF (250 mg q12h) was resumed (Fig. 2). The patient returned home on day 69 of illness. To date, he has been in good health at home for 4 months. 


\section{Cardiorenal Medicine}

\begin{tabular}{l|l}
\hline Cardiorenal Med 2020;10:470-475 \\
\hline DOI: 10.1159/000510916 & $\begin{array}{l}\text { @ 2020 S. Karger AG, Basel } \\
\text { www.karger.com/crm }\end{array}$ \\
\hline
\end{tabular}

Zhu et al.: Recovery from COVID-19 in a Renal Transplant Recipient with Acute Cardiorenal Syndrome

\section{Discussion}

Acute kidney injury (AKI) has been reported in 25-27.8\% of patients with severe or critical COVID-19 pneumonia [3-5]. Renal transplant recipients with COVID-19 pneumonia may be more susceptible to AKI due to a potential lack of renal function [2]. In this case, a reduced urine output and a progressive increase in serum creatinine and BUN were prominent early signs of the disease. In particular, after the onset of atrial fibrillation, acute myocardial injury, and heart failure, the patient's renal function nearly collapsed, suggesting the occurrence of acute cardiorenal syndrome. After correction of the arrhythmia and heart failure, the patient recovered quickly from the AKI after intensive diuretic treatment and strict control of fluid intake. This result indicates that abnormal cardiac function is closely related to the severity of AKI, and improving cardiac function is critical for recovery from AKI. Since most kidney transplant patients have a long history of hypertension and underlying heart disease, it is necessary to pay close attention to protection of the cardiovascular system in the treatment of COVID-19.

When treating pneumonia due to virus infection following organ transplantation, the adjustment of immunosuppressive agents is a common strategy and allows recipients the opportunity to reacquire anti-infection immunity within a short period, which is conducive to eliminating the virus [6]. Considering the absence of fever at the onset of illness in this case, the patient's oral immunosuppressants were not reduced in the early stage of COVID-19. Until the course of the disease was close to 3 weeks, when the nucleic acid test for SARS-Cov- 2 was still positive and the lung CT showed a slow absorption of inflammation, the patient's immunosuppressants began to be reduced. In addition, thymosin and IVIG were given to accelerate the recovery of the patient's immunity. Other therapeutic measures included antiviral treatment (Arbidol), prevention of secondary bacterial/fungal infections (moxifloxacin, meropenem, and micafungin), and adjuvant therapy.

The antibody response to SARS-Cov-2 may facilitate virus clearance. In this case, the patient developed a delayed and lower level of virus-specific IgG $(40.7 \mathrm{AU} / \mathrm{mL})$ compared to what has been reported for the general population (median: 161.2 AU/mL) [7]. This result suggest that immunosuppression may have a significant impact on the antibody response against the SARS-Cov-2 virus. Interestingly, after the patient was discharged from the hospital after 2 consecutive negative tests for SARS-Cov-2, he subsequently and repeatedly tested positive again for the virus. Since the patient's oral immunosuppressants were not reduced until the course of COVID-19 had progressed nearly 3 weeks, we speculate that the slow virus clearance in this patient may be related to his long-term history of immunosuppression and delayed adjustment of the immunosuppressants during the COVID-19.

It has become a recurring and puzzling phenomenon that patients whose respiratory nucleic acid tests for SARS-Cov-2 are negative at least twice then test positive again after discharge [8]. In this case, even though the patient tested positive for the virus twice after discharge, the COVID-19 pneumonia showed no signs of worsening and continued to improve. This suggests that the reappearance of positive tests for SARS-Cov-2 in the late stage of recovery may not represent a relapse of the disease.

We reported the clinical features and therapeutic course of COVID-19 pneumonia in a renal transplant patient with early severe cardiorenal syndrome. Although the patient underwent a relatively slow process of virus clearance, he recovered from COVID-19 pneumonia after timely correction of heart and kidney function, thus providing a reference case for treating such patients. 


\section{Cardiorenal Medicine}

\begin{tabular}{l|l}
\hline Cardiorenal Med 2020;10:470-475 \\
\hline DOI: 10.1159/000510916 & $\begin{array}{l}\text { @ 2020 S. Karger AG, Basel } \\
\text { www.karger.com/crm }\end{array}$ \\
\hline
\end{tabular}

Zhu et al.: Recovery from COVID-19 in a Renal Transplant Recipient with Acute Cardiorenal Syndrome

\section{Acknowledgement}

We thank Dr. Ke Ma from the Department of Infectious Disease, Tongji Hospital, Tongji Medical College, Huazhong University of Science and Technology, who provided insight and expertise that greatly assisted this research. We thank Mr. Zhiliang Guo for assistance with data collection.

\section{Statement of Ethics}

This study was approved by the institutional review board (IRB) of Tongji Hospital, Tongji Medical College, Huazhong University of Science and Technology (IRB approval No.: TJ-C20200120). Written informed consent was waived for emerging infectious disease, but verbal informed consent was obtained. This study was conducted in accordance with the principles of the Declaration of Helsinki.

\section{Conflict of Interest Statement}

The authors have no conflict of interests to declare.

\section{Funding Source}

This study was supported by the Non-Profit Central Research Institute Fund of the Chinese Academy of Medical Science (grant No. 2018PT32018).

\section{Author Contributions}

G.C. made substantial contributions to the study concept and substantial revisions to this paper. L.Z. participated in obtaining ethical approval, collecting data, confirming data accuracy, and drafting this paper. H.S. was responsible for CT images acquisition and interpretation. H.L. contributed to laboratory testing. T.Q. and J.Z. contributed to the treatment of subjects and data acquisition.

\section{References}

1 Akalin E, Azzi Y, Bartash R, Seethamraju H, Parides M, Hemmige V, et al. Covid-19 and Kidney Transplantation. N Engl J Med. 2020 Jun;382(25):2475-7.

2 Zhu L, Gong N, Liu B, Lu X, Chen D, Chen S, Shu H, Ma K, Xu X, Guo Z et al: Coronavirus disease 2019 pneumonia in immunosuppressed renal transplant recipients: a summary of 10 confirmed cases in Wuhan, China. Eur Urol. 2020 Jun;77(6):748-54.

3 Fanelli V, Fiorentino M, Cantaluppi V, Gesualdo L, Stallone G, Ronco C, et al. Acute kidney injury in SARS-CoV-2 infected patients. Crit Care. 2020 Apr;24(1):155.

4 Yang X, Yu Y, Xu J, Shu H, Xia J, Liu H, et al. Clinical course and outcomes of critically ill patients with SARSCoV-2 pneumonia in Wuhan, China: a single-centered, retrospective, observational study. Lancet Respir Med. 2020 May;8(5):475-81.

5 Zhou F, Yu T, Du R, Fan G, Liu Y, Liu Z, et al. Clinical course and risk factors for mortality of adult inpatients with COVID-19 in Wuhan, China: a retrospective cohort study. Lancet. 2020 Mar;395(10229):1054-62.

6 Kidney Disease: Improving Global Outcomes Transplant Work Group: KDIGO clinical practice guideline for the care of kidney transplant recipients. Am J Transplant. 2009;9 Suppl 3:S1-155.

7 Jin Y, Wang M, Zuo Z, Fan C, Ye F, Cai Z, et al. Diagnostic value and dynamic variance of serum antibody in coronavirus disease 2019. Int J Infect Dis. 2020 May;94:49-52.

8 Lan L, Xu D, Ye G, Xia C, Wang S, Li Y, et al. Positive RT-PCR Test Results in Patients Recovered From COVID-19. JAMA. 2020 Apr;323(15):1502-3. 